\title{
ON FREE SPECTRA OF A CLASS OF FINITE INVERSE MONOIDS
}

\author{
IGOR DOLINKA \\ (Received 19 July 2011; accepted 26 October 2011; first published online 7 March 2013) \\ Communicated by M. G. Jackson

\begin{abstract}
For a finite Clifford inverse algebra $A$, with natural order meet-semilattice $Y_{A}$ and group of units $G_{A}$, we show that the inverse monoid obtained as the semidirect product $Y_{A}^{1} *_{\rho} G_{A}$ has a $\log$-polynomial free spectrum whenever $\rho$ is a term-expressible left action of $G_{A}$ on $Y_{A}$ and all subgroups of $A$ are nilpotent. This yields a number of examples of finite inverse monoids satisfying the Seif conjecture on finite monoids whose free spectra are not doubly exponential.
\end{abstract}

2010 Mathematics subject classification: primary 20M05; secondary 20M18, $08 \mathrm{~B} 20$.

Keywords and phrases: free spectrum, finite inverse monoid, semidirect product, Clifford inverse algebra.

\section{Introduction}

Let $\mathcal{V}$ be a locally finite variety, in the sense of universal algebra [3]. The free spectrum $f_{n}(\mathcal{V})$ of $\mathcal{V}$ is the sequence consisting of the cardinalities of its $n$-generated free algebras [9]. In particular, if $A$ is a finite algebra, the free spectrum of $A$ is simply the free spectrum of the variety $\mathcal{V}(A)$ it generates. The free spectrum is an important invariant of an algebra generating a locally finite variety that is intimately related to a range of its structural properties. One of the most interesting properties in this regard is the rate of growth of $f_{n}(\mathcal{V})$, that is, its asymptotic behavior. We say that a variety $V$ has at least a doubly exponential free spectrum if there is a positive real number $c$ such that

$$
f_{n}(\mathcal{V}) \geq 2^{2^{c n}}
$$

holds for large $n$. This is precisely as far as finitely generated varieties can go, since elementary universal-algebraic arguments imply that for a finite algebra $A$ we have $f_{n}(A) \leq|A|^{|A|^{n}}$. A strikingly different behavior of a free spectrum is encountered

The research of the author is supported by the Ministry of Education and Science of the Republic of Serbia through Grant No. 174019, and by a grant (Contract 114-451-2002/2011) of the the Secretariat of Science and Technological Development of the Autonomous Province of Vojvodina.

(C) 2013 Australian Mathematical Publishing Association Inc. 1446-7887/2013 \$16.00 
when $f_{n}(\mathcal{V})$ is log-polynomial, which means that $\log f_{n}(\mathcal{V})$ is bounded above by a polynomial function in $n$ (where 'log' refers to the base-2 logarithm).

The fact that a finite algebra has a doubly exponential free spectrum indicates that it is sufficiently close to being functionally complete, since it has a rich clone of term/polynomial operations. For example, the free spectrum of the variety of Boolean algebras is precisely $2^{2^{n}}$, and even the two-element lattice (generating the variety of distributive lattices) has a doubly exponential free spectrum. The same is true for any nonnilpotent finite group [20]. The results of [1] and [11, Chapter 12] offer several universal-algebraic generalisations of these classical results. On the other hand, if a finite group $G$ is nilpotent of class $c$, then $\log f_{n}(G)$ belongs to the asymptotic class $O\left(n^{c}\right)$ (see Higman [10] and Neumann [20]).

One of the most important discoveries concerning free spectra was made by Kearnes [16], who observed that the asymptotic behavior of a finite general algebra $A$ is largely governed by the free spectrum of a naturally associated monoid $\operatorname{Tw}(A)$, called the twin monoid. This provided a significant boost for the investigation of free spectra of finite monoids and semigroups, and, in particular, for finding the watershed between log-polynomial and doubly exponential ones (and determining if there is anything in between). A pioneering paper in this vein was recently written by Seif [24], who conjectured that a finite monoid $M$ does not have a doubly exponential free spectrum if and only if $M$ has only nilpotent subgroups and belongs to a certain pseudovariety (a class of finite monoids closed under taking homomorphic images, submonoids and finite direct products) called EDA, which is, incidentally, a well-studied object in finite semigroup theory. (Namely, the pseudovariety DA comprises finite monoids all of whose regular $\mathscr{D}$-classes are rectangular bands, and a finite monoid belongs to EDA if and only if its idempotent generated submonoid belongs to DA.) Seif himself proved the forward implication of his conjecture and verified its converse for monoids obtained by adjoining an identity element to a finite Rees matrix (that is, completely 0-simple) semigroup. Subsequently, the author in [5] confirmed the Seif conjecture for completely regular monoids (monoids that are unions of their subgroups): more generally, a completely regular semigroup has a sub-log-exponential (in fact, a log-polynomial) free spectrum if and only if it is locally orthodox and all of its subgroups are nilpotent. Also, all monoids in DA are shown in [7] to have a logpolynomial free spectrum. Some other related results concerning free spectra of semigroups may be found, for example, in [4, 6, 14, 15].

The next important particular instance of the Seif conjecture-and, in a sense, a real 'test case' for it-concerns inverse monoids [18]. Namely, since the idempotents of any inverse semigroup form a semilattice under multiplication, all finite inverse monoids belong to EDA. Therefore, a verification of the conjecture in the inverse case would consist of proving that each finite inverse monoid with nilpotent subgroups has a free spectrum that is not doubly exponential (perhaps even log-polynomial). In the present state of knowledge on finitely generated inverse semigroup varieties, this appears to be a distant prospect. Yet, in this note we present a reasonably broad class of finite inverse monoids with log-polynomial free spectra. These inverse 
monoids are obtained from the so-called (Clifford) inverse algebras extensively studied by Leech in [19]; loosely speaking, an inverse algebra arises whenever the natural partial order that can be defined on any inverse monoid turns out to be a meetsemilattice order, which results in a semiring-like algebraic structure. So, we take this semilattice component of a Clifford inverse algebra $A$ (see Section 2.3 below) and construct its semidirect product by $G_{A}$, the group of units of $A$, with respect to a (term-definable) left action of this group on the meet-semilattice reduct $Y_{A}$ of $A$; we argue (in Theorem 2.7 below, our main result) that any such semidirect product-and thus any inverse semigroup belonging to the variety it generates-has a free spectrum whose logarithm is bounded above by a polynomial. We exhibit a few examples of finite inverse monoids which are covered by this construction. In particular, we will recover a polynomial upper bound for $\log f_{n}\left(B_{2}^{1}\right)$, where $B_{2}^{1}$ is the six-element Brandt monoid, which presented the main obstacle in [24] in obtaining log-polynomial upper bounds for monoids obtained from finite completely 0 -simple semigroups over nilpotent groups.

\section{Preliminaries and formulation of the main result}

2.1. Inverse semigroups. A semigroup $S$ is (von Neumann) regular if for each $a \in S$ there is an $x \in S$ such that $a x a=a$. If, in addition, $x a x=x$, then $x$ is called an inverse of $a$. A regular semigroup is inverse if its idempotent elements commute (and so form a semilattice); it is not difficult to see that an equivalent condition is that every element has a unique inverse (we refer to [18] for further background in general and inverse semigroup theory). It is this latter definition that offers the possibility to define inverse semigroups as an equational class in the extended signature, which, beyond the binary multiplication symbol, contains a unary symbol ${ }^{-1}$. The associative law and the identities

$$
(x y)^{-1} \approx y^{-1} x^{-1}, \quad\left(x^{-1}\right)^{-1} \approx x, \quad x x^{-1} x \approx x, \quad x x^{-1} y y^{-1} \approx y y^{-1} x x^{-1}
$$

ensure that $a^{-1}$ is always the unique inverse of $a$. An inverse monoid is an inverse semigroup that possesses an identity element; by adding the constant symbol 1 and the identities $x 1 \approx 1 x \approx x$ and $1^{-1} \approx 1$ we establish that inverse monoids form a variety, too.

On the other hand, inverse semigroups (or monoids) in the signature consisting only of the binary symbol (and, perhaps, the constant 1) do not form a variety, and so if $S$ is an inverse semigroup/monoid, the variety generated by it may well contain noninverse semigroups. Therefore, the varieties generated by an inverse monoid in the monoid signature and in the extended signature are different, as well as their free objects and, consequently, free spectra. Nevertheless, there is a strong connection.

Lemma 2.1. Let $S$ be an inverse monoid generating a locally finite monoid variety, and let $f_{n}$ and $f_{n}^{\prime}$ be its free spectra when considered as a plain monoid and as an inverse monoid with the unary inverse operation, respectively. Then $f_{n} \leq f_{n}^{\prime} \leq f_{2 n}$ for all positive integers $n$. 
Proof. It is well known in universal algebra that the standard model of the $n$-generated free algebra in the variety generated by $A$ consists of all term operations of $A$ over a fixed set of $n$ variables. Hence, $f_{n}(A)$ coincides with the number of $n$-ary term operations of $A$. Since the term operations of the plain monoid $S$ are just operations induced by (possibly empty) words, any such operation is at the same time a term operation of $S$ considered as an inverse monoid; therefore, $f_{n} \leq f_{n}^{\prime}$.

On the other hand, the identities $(x y)^{-1} \approx y^{-1} x^{-1}$ and $\left(x^{-1}\right)^{-1} \approx x$ imply that any term operation of the inverse monoid $S$ over an $n$-element set of variables $X_{n}=\left\{x_{1}, \ldots, x_{n}\right\}$ is equivalent to the operation induced by a word $\mathbf{w}\left(x_{1}, \ldots, x_{n}, x_{1}^{-1}, \ldots, x_{n}^{-1}\right)$ over the 'doubled' alphabet $X_{n} \cup X_{n}^{-1}$. In fact, the latter word can be considered as a substitution instance of a plain word $\mathbf{w}$ over a $2 n$-element alphabet, obtained by replacing each occurrence of $x_{i}^{-1}$ by a new letter, say $y_{i}$. Then, clearly, if the inverse monoid identity $\mathbf{u}\left(x_{1}, \ldots, x_{n}, x_{1}^{-1}, \ldots, x_{n}^{-1}\right) \approx \mathbf{v}\left(x_{1}, \ldots, x_{n}, x_{1}^{-1}, \ldots, x_{n}^{-1}\right)$ fails in $S$, so does the plain monoid identity $\mathbf{u} \approx \mathbf{v}$. This shows that $f_{n}^{\prime} \leq f_{2 n}$.

The previous lemma shows that there is no harm in considering all the inverse monoids in the remainder of this paper to be algebras of the form $\left(S, \cdot,{ }^{-1}, 1\right)$ of the type $(2,1,0)$ and to identify their free spectra with the number of operations induced on $S$ by inverse monoid words.

2.2. Semidirect product of a semilattice by a group. A theoretically very important construction of inverse semigroups is that of a semidirect product of a semilattice by a group. Namely, let $Y$ be a meet-semilattice monoid, whose binary operation is denoted by $\wedge$, considered as an inverse monoid (the unary operation ${ }^{-1}$ is just the identity mapping). Furthermore, let $G$ be a group acting by automorphisms on $Y$; we write this (left) action as $\rho:(g, \alpha) \mapsto g \cdot \alpha$, where $\alpha \in Y$ and $g \in G$. In effect, we have a homomorphism $\rho: G \rightarrow \operatorname{Aut}(Y)$. The semidirect product of $Y$ by $G$ with respect to $\rho$ is the inverse monoid $Y *{ }_{\rho} G$ defined on $Y \times G$ by

$$
(\alpha, g)(\beta, h)=(\alpha \wedge g \cdot \beta, g h) \text { and }(\alpha, g)^{-1}=\left(g^{-1} \cdot \alpha, g^{-1}\right)
$$

for all $\alpha, \beta \in Y$ and $g, h \in G$. The significance of this construction is underlined by the following basic result in the theory of inverse semigroups: every (finite) inverse semigroup/monoid $S$ is a homomorphic image of a subalgebra of a (finite) semidirect product $Y *_{\rho} G$ for suitable $\rho$ and (finite) $Y$ and $G$. In fact, $Y$ can be taken to be the semilattice $E(S)$. See $[8,18]$ for additional background.

2.3. (Clifford) inverse algebras. It is well known-and, indeed, one of the main motifs in inverse semigroup theory-that the operations of an inverse semigroup $S$ induce a natural order $\leq$ on $S$ defined for $a, b \in S$ by

$$
a \leq b \text { if and only if } a=b a^{-1} a .
$$

The natural order is stable under left and right multiplication. One of the most interesting situations occurs when the natural order is a meet-semilattice order, and such a situation allows an additional binary operation $\wedge$ to be defined on $S$ (as is done 
by several authors), inducing a semilattice order identical to the natural one. Following Leech [19], we call inverse monoids equipped with such an operation inverse algebras. It was shown in $[13,19]$ that inverse algebras form a variety, and that it is defined by: inverse monoid axioms for $\cdot,^{-1}$ and 1 , the semilattice axioms for $\wedge$, both distributive laws for $\cdot$ over $\wedge$, and the identity

$$
x \wedge y \approx x(x \wedge y)^{-1}(x \wedge y)
$$

A Clifford monoid is an inverse monoid in which all idempotents are central (or, equivalently, which can be decomposed into a semilattice of its maximal subgroups). If the underlying inverse monoid of an inverse algebra is Clifford, then the algebra in question is called a Clifford inverse algebra.

Two main examples of Clifford inverse algebras follow.

Example 2.2. For a group $G$, define an algebra on $G \cup\{\infty\}$ (where $\infty \notin G$ ) by setting $\infty^{2}=\infty^{-1}=\infty$ and $\infty g=g \infty=\infty$ and expanding the obtained inverse monoid by a meet semilattice operation $\wedge$ such that $\infty \wedge g=\infty$ and $g \wedge h=\infty$ whenever $g \neq h$. The resulting algebra is denoted by $b(G)$ and called the sink algebra over $G$ (also called in [12] the flat extension of $G$ ). As is easily verified, $b(G)$ is always a Clifford inverse algebra.

ExAmple 2.3 [19, Paragraph 3.12]. Let $S$ be an arbitrary Clifford monoid. Then $N(S)$ is defined to be the Clifford inverse algebra consisting of all cosets of all normal subgroups of all maximal subgroups of $S$ : if $G_{x}$ is the maximal subgroup containing $x \in S$, then $N x$ is a typical example of such a coset, $N \unlhd G_{x}$. The multiplication works as follows: $K x \cdot H y=N x y$, where $N$ is the least normal subgroup of $G_{x y}$ containing $K y y^{-1} \cup H x x^{-1}$. The natural semilattice order yields $K x \leq H y$ if and only if $K x \supseteq H y$; thus $K x \wedge H y$ is the least coset of a normal subgroup of $G$ containing $K x \cup H y$ (it is easily verified that such a least coset always exists). The inverse monoid $S$ itself embeds into $N(S)$ via $x \mapsto E x=\{x\}$. In that sense, $S$ (that is, the singletons) generates a subalgebra of $N(S)$, which is denoted by $N^{*}(S)$ : it consists of all cosets of finitely generated normal subgroups of maximal subgroups of $S$. Hence, we have $N(S)=N^{*}(S)$ whenever the Clifford monoid $S$ is finite.

Notice that if $S$ is a group, then $b(S)$ is just a quotient of $N(S)$ obtained from the congruence collapsing all nonsingleton cosets of $S$.

Here are the existing results that we will utilise in what follows.

FАСт 2.4 (see [12, Theorem 7.5]). Clifford inverse algebras form a variety, $\mathcal{C}$, which is generated by sink algebras b $(G)$ of all groups $G$. Furthermore, for each group variety $\mathcal{V}$, the class $C(\mathcal{V})$ of all Clifford inverse algebras all of whose subgroups belong to $\mathcal{V}$ is a subvariety of $C$.

FACT 2.5 (see [19, Paragraph 3.17 and Theorem 3.13]). Let $\mathcal{V}$ be a variety of groups, and let $\mathcal{V}^{\text {sl }}$ denote the variety of Clifford inverse monoids all of whose subgroups 
belong to $\mathcal{V}$. If $S$ is the free object of $\mathcal{V}^{\mathrm{sl}}$ on $n>0$ free generators, then $\boldsymbol{N}^{*}(S)$ is isomorphic to $F_{n}(C(\mathcal{V}))$, the n-generated free object of $C(\mathcal{V})$.

2.4. The main theorem. Let $\left(A, \wedge, \cdot,{ }^{-1}, 1\right)$ be a Clifford inverse algebra. We denote by $Y_{A}$ its semilattice reduct $(A, \wedge)$, and, as is customary in semigroup theory, by $Y_{A}^{1}$ the monoid obtained by adjoining an identity element to $Y_{A}$ unless it already has one. Denote by $G_{A}$ the group of units of $A$, that is, the maximal subgroup of $A$ containing the identity element 1 .

There are several ways in which $G_{A}$ may act on the semilattice $Y_{A}$ by automorphisms; for example, here is one.

Lemma 2.6. Let $A$ be a Clifford inverse algebra and let $g \in G_{A}$. Then the left multiplication by $g, \lambda_{g}: a \mapsto g a, a \in A$, is an automorphism of $Y_{A}$.

Proof. By left distributivity of multiplication over the meet operation in inverse algebras, we have $\lambda_{g}(a \wedge b)=g(a \wedge b)=g a \wedge g b=\lambda_{g}(a) \wedge \lambda_{g}(b)$ for all $g \in G_{A}$ and $a, b \in Y_{A}$, so that each $\lambda_{g}$ is a semilattice endomorphism of $Y_{A}$. Obviously, $\lambda_{g}\left(g^{-1} a\right)=$ $a$, thus $\lambda_{g}$ is surjective. To prove that $\lambda_{g}$ is injective, we recall a basic fact on Clifford semigroups/monoids (see [18, Theorem 5.2.12]): any such semigroup can be represented as a strong semilattice of its maximal subgroups. In more detail, we have a family of groups $\left\{G_{\alpha}: \alpha \in Y\right\}$ indexed by a semilattice $Y$ (or a semilattice monoid, if we work with monoids). Along with this family comes a system of group homomorphisms $\phi_{\alpha, \beta}: G_{\alpha} \rightarrow G_{\beta}$ for any $\alpha, \beta \in Y$ such that $\alpha \geq \beta$, subject to the following two conditions: (1) $\phi_{\alpha, \alpha}$ is the identity mapping on $G_{\alpha}$ for any $\alpha \in Y$; and (2) for any $\alpha, \beta, \gamma \in Y$ such that $\alpha \geq \beta \geq \gamma$ we have $\phi_{\alpha, \gamma}=\phi_{\beta, \gamma} \phi_{\alpha, \beta}$. The multiplication in the strong semilattice of groups works as follows: if $g \in G_{\alpha}$ and $h \in G_{\beta}$, then $g h=\phi_{\alpha, \alpha \wedge \beta}(g) \phi_{\beta, \alpha \wedge \beta}(h)$, the right-hand side being a product within the group $G_{\alpha \wedge \beta}$. Therefore, the monoid reduct of $A$ is constructed from a suitable system of groups and group homomorphisms. In particular, if $Y$ is the structure semilattice of that monoid reduct, then $G_{A}=G_{\varepsilon}$, where $\varepsilon$ is the identity element of $Y$. Thus if we assume that $g a=g b$ for some $a, b \in A$ and $g \in G_{A}$ such that $a \in G_{\alpha}, b \in G_{\beta}$ for some $\alpha, \beta \in Y$, we immediately conclude that $\alpha=\beta$. Hence, $g a=\phi_{\varepsilon, \alpha}(g) \phi_{\alpha, \alpha}(a)=\phi_{\varepsilon, \alpha}(g) a$ and, similarly, $g b=\phi_{\varepsilon, \alpha}(g) b$, so $g a=g b$ implies $a=b$, as required.

Finally, notice that $\lambda_{g} \lambda_{h}=\lambda_{g h}$ and that $\lambda_{1}$ is the identity mapping on $Y_{A}$, so that we indeed have a left action of $G_{A}$ on the semilattice $Y_{A}$.

We can extend the automorphism $\lambda_{g}$ of $Y_{A}$ to an automorphism $\lambda_{g}^{\prime}$ of $Y_{A}^{1}$ by fixing its adjoined top element, which we denote by $\top$ (to distinguish from 1, the identity element of the algebra $A$, which is one level 'lower' in $Y_{A}^{1}$ ). Similarly, we may consider the action of $G_{A}$ on $Y_{A}$ by conjugation in $A$, so that $g \cdot a=g a g^{-1}$ for $g \in G_{A}$ and $a \in A$, and extend it to an action on $Y_{A}^{1}$ by fixing $\mathrm{T}$. What is common in these actions is that $g \cdot a$ can be expressed as $t(g, a)$ for a binary term operation $t(x, y)$ of the algebra $A$. We will call such actions term-expressible. 
Now, with respect to a fixed term-expressible action $\rho:(g, a) \mapsto t(g, a)$ we can form the semidirect product $Y_{A}^{1} *_{\rho} G_{A}$ of $Y_{A}^{1}$ and $G_{A}$. The following is the main result of this paper.

Theorem 2.7. Let $A$ be a Clifford inverse algebra such that the set of all of its subgroups generate a locally finite group variety $\mathcal{V}$, and let $\rho$ be a term-expressible left action of $G_{A}$ on $Y_{A}^{1}$. Then

$$
\log f_{n}\left(Y_{A}^{1} *_{\rho} G_{A}\right) \in \mathscr{O}\left(n\left(\log f_{n+1}(\mathcal{V})\right)^{2}\right) .
$$

By a direct application of the Higman-Neumann result, we obtain the following consequence.

Corollary 2.8. If $A$ is a finite Clifford inverse algebra such that all of its subgroups are nilpotent of class $c$, while $\rho$ is a term-expressible left action of $G_{A}$ on $Y_{A}^{1}$, then

$$
\log f_{n}\left(Y_{A}^{1} *_{\rho} G_{A}\right) \in \mathscr{O}\left(n^{2 c+1}\right) .
$$

Consequently, the same is true for any finite inverse semigroup belonging to the variety generated by $Y_{A}^{1} *_{\rho} G_{A}$.

\section{Proof of Theorem 2.7}

The vehicle in establishing our principal result is the following inequality.

Proposition 3.1. Let A be a Clifford inverse algebra such that both $A$ and $G_{A}$ generate locally finite varieties (of inverse algebras and groups, respectively), and let $\rho$ be a term-expressible left action of $G_{A}$ on $Y_{A}^{1}$. Then

$$
f_{n}\left(Y_{A}^{1} *_{\rho} G_{A}\right) \leq f_{n}\left(G_{A}\right)\left(f_{n+1}(A)\right)^{n} .
$$

PROOF. First of all, we look at the way of representing an arbitrary inverse monoid word $\mathbf{w}=y_{1} \cdots y_{m} \in\left(X_{n} \cup X_{n}^{-1}\right)^{*}$ (over an $n$-element alphabet $X_{n}$ ) in a general semidirect product $Y *{ }_{\rho} G$ of a semilattice monoid $Y$ by a group $G$ with respect to an arbitrary left action $\rho:(g, \alpha) \mapsto g \cdot \alpha$, where $\alpha \in Y, g \in G$. To this end, we substitute each variable $x_{j} \in X_{n}$ by a pair of variables $\left(\xi_{j}, \gamma_{j}\right)$ taking values in $Y$ and $G$, respectively; then $x_{j}^{-1}$ becomes $\left(\gamma_{j}^{-1} \cdot \xi_{j}, \gamma_{j}^{-1}\right)$. Now, respecting the way the multiplication works in $Y *_{\rho} G$, aided by the group action $\rho$ and the semilattice operation $\wedge$, this substitution yields

$$
\mathbf{w}\left(\left(\xi_{1}, \gamma_{1}\right), \ldots,\left(\xi_{n}, \gamma_{n}\right)\right)=\left(\bigwedge_{i=1}^{m} E_{i}, \mathbf{w}\left(\gamma_{1}, \ldots, \gamma_{n}\right)\right)
$$

where the expressions $E_{i}$ will be explained in the following, while $\mathbf{w}\left(\gamma_{1}, \ldots, \gamma_{n}\right)$ is simply a group word over the alphabet $\left\{\gamma_{1}, \ldots, \gamma_{n}\right\}$. So, let $\mathbf{w}_{i}=y_{1} \cdots y_{i}$ be a prefix of $\mathbf{w}$ of length $i \geq 0$ ( $\mathbf{w}_{0}$ is the empty word). When $i>0$, we distinguish two cases:

- $y_{i}=x_{j_{i}} \in X_{n}$. Then $E_{i}=\mathbf{w}_{i-1}\left(\gamma_{1}, \ldots, \gamma_{n}\right) \cdot \xi_{j_{i}}$.

- $y_{i}=x_{j_{i}}^{-1} \in X_{n}^{-1}$. In this case $E_{i}=\mathbf{w}_{i-1}\left(\gamma_{1}, \ldots, \gamma_{n}\right) \gamma_{j_{i}}^{-1} \cdot \xi_{j_{i}}$. 
If we agree, for convenience, to write $(g \wedge h) \cdot \alpha$ instead of $g \cdot \alpha \wedge h \cdot \alpha$, then $\bigwedge_{i=1}^{m} E_{i}$ can be written as a 'linear combination'

$$
\bigwedge_{j=1}^{n} C_{j} \cdot \xi_{j},
$$

where each 'coefficient' $C_{j}$ is a finite 'meet' of some group words over $\left\{\gamma_{1}, \ldots, \gamma_{n}\right\}$.

Let us now turn to the case where $Y=Y_{A}^{1}$ and $G=G_{A}$ acts on $Y$ by a left action $\rho$ associated with an inverse algebra term $\mathbf{t}(\mathrm{x}, \mathrm{y})$. The previous analysis shows that if $\mathbf{w}, \mathbf{w}^{\prime}$ are two words over $\left(X_{n} \cup X_{n}^{-1}\right)^{*}$, then the identity $\mathbf{w} \approx \mathbf{w}^{\prime}$ fails in $Y_{A}^{1} *_{\rho} G_{A}$ if and only if it either fails in $G_{A}$, or $\bigwedge_{j=1}^{n} C_{j} \cdot \xi_{j} \approx \bigwedge_{j=1}^{n} C_{j}^{\prime} \cdot \xi_{j}$ fails for the 'linear combinations' that $\mathbf{w}$ and $\mathbf{w}^{\prime}$ induce on $Y_{A}^{1}$, respectively.

CLAIM. If the semidirect product $Y_{A}^{1} *_{\rho} G_{A}$ fails to satisfy $\bigwedge_{j=1}^{n} C_{j} \cdot \xi_{j} \approx \bigwedge_{j=1}^{n} C_{j}^{\prime} \cdot \xi_{j}$, then the identity $\mathbf{t}\left(\mathrm{C}_{j}, \xi\right) \approx \mathbf{t}\left(\mathrm{C}_{j}^{\prime}, \xi\right)$ fails in $A$ for some $1 \leq j \leq n$.

To see this, first of all, by substituting the top element $\mathrm{T}$ of $Y_{A}^{1}$ for all but one of the semilattice variables $\xi_{j}$, we see that $\bigwedge_{j=1}^{n} C_{j} \cdot \xi_{j} \approx \bigwedge_{j=1}^{n} C_{j}^{\prime} \cdot \xi_{j}$ holds in $Y_{A}^{1} *_{\rho} G_{A}$ if and only if

$$
C_{j}\left(\gamma_{1}, \ldots, \gamma_{n}\right) \cdot \xi_{j} \approx C_{j}^{\prime}\left(\gamma_{1}, \ldots, \gamma_{n}\right) \cdot \xi_{j}
$$

holds for each $1 \leq j \leq n$. Clearly, (3.1) is satisfied if $\xi_{j}=\top$ regardless of the form of the coefficients $C_{j}, C_{j}^{\prime}$. Therefore, there is an index $j$ such that (3.1) fails for $\xi_{j}$ evaluated as an element of $\alpha \in Y_{A}$, and for each $\gamma_{r}, 1 \leq r \leq n$, evaluated as some $g_{r} \in G_{A}$. In other words, if $t, \widehat{C}_{j}, \widehat{C}_{j}^{\prime}$ are the term operations of $A$ induced by the terms $\mathbf{t}, C_{j}, C_{j}^{\prime}$, respectively, then

$$
t\left(\widehat{C}_{j}\left(g_{1}, \ldots, g_{n}\right), \alpha\right)=t\left(\widehat{C}_{j}^{\prime}\left(g_{1}, \ldots, g_{n}\right), \alpha\right)
$$

Hence, $\mathbf{t}\left(\mathrm{C}_{j}, \xi\right) \approx \mathbf{t}\left(\mathrm{C}_{j}^{\prime}, \xi\right)$ fails in $A$. This establishes our claim.

In conclusion, to each $n$-ary term operation on $Y_{A}^{1} *_{\rho} G_{A}$ induced by an inverse monoid word $\mathbf{w}$ we can associate an $(n+1)$-tuple

$$
\left(t\left(\widehat{C}_{1}(\bar{x}), y\right), \ldots, t\left(\widehat{C}_{n}(\bar{x}), y\right), \widehat{\mathbf{w}}(\bar{x})\right)
$$

(where $\bar{x}=\left(x_{1}, \ldots, x_{n}\right)$ and $\widehat{\mathbf{w}}$ is the term operation of $G_{A}$ induced by $\mathbf{w}$ ) consisting of $n$ term operations of $A$ (on $n+1$ variables) and an $n$-ary term operation of $G$. The previous claim and the considerations preceding it show that such a mapping is injective, so the proposition follows.

Proof of Theorem 2.7. We start by recalling the well-known fact that the structure semilattice (the greatest semilattice homomorphic image) of $S=F_{n}\left(\mathcal{V}^{\mathrm{sl}}\right)$, the $n$ generated relatively free Clifford monoid of $\mathcal{V}^{\text {sl }}$, is $\mathcal{P}\left(X_{n}\right)$, the $\cap$-semilattice of all subsets of an $n$-element set $X_{n}$, while for $Z \subseteq X_{n}$ the corresponding maximal subgroup $G_{Z}$ (whose identity is $e_{Z}=\prod_{z \in Z} z z^{-1}$ ) is isomorphic to the $\mathcal{V}$-free group $F_{|Z|}(\mathcal{V})$. 
By the conditions given, $A \in C(\mathcal{V})$. So, from Fact 2.5 we have the following chain of inequalities:

$$
f_{n}(A) \leq\left|F_{n}(C(\mathcal{V}))\right| \leq\left|N^{*}(S)\right| \leq|N(S)|=\sum_{k=0}^{n}\left(\begin{array}{l}
n \\
k
\end{array}\right) \sum_{N \unlhd F_{k}(\mathcal{V})}\left[F_{k}(\mathcal{V}): N\right]
$$

The final touch is provided by the seminal memoir of Berman and Idziak [2] on generative complexity of (finite) algebras: namely, their Lemma 6.8 provides an upper bound on the size of the congruence lattice of a finite congruence-uniform algebra (which means that for any congruence $\theta$ all $\theta$-blocks have the same size).

FАСТ 3.2 [2]. If B is a finite, congruence-uniform algebra, then

$$
|\operatorname{Con}(B)| \leq|B|^{2 \log |B|} .
$$

Since $\mathcal{V}$ is locally finite, $F_{k}(\mathcal{V})$ is a finite group for any finite $k$, a congruenceuniform algebra (the congruence blocks are just cosets of a normal subgroup). Therefore, the number of normal subgroups of $F_{n}(\mathcal{V})$ is estimated by

$$
\log \left|\operatorname{Con}\left(F_{n}(\mathcal{V})\right)\right| \leq 2\left(\log \left|F_{n}(\mathcal{V})\right|\right)^{2}=2\left(\log f_{n}(\mathcal{V})\right)^{2} .
$$

Thus the previous chain of inequalities continues as follows:

$$
f_{n}(A) \leq \sum_{k=0}^{n}\left(\begin{array}{l}
n \\
k
\end{array}\right) \sum_{N \unlhd F_{k}(\mathcal{V})}\left[F_{k}(\mathcal{V}): N\right] \leq 2^{n} \cdot\left|F_{n}(\mathcal{V})\right| \cdot\left|\operatorname{Con}\left(F_{n}(\mathcal{V})\right)\right|,
$$

implying that

$$
\log f_{n}(A) \leq n+\log f_{n}(\mathcal{V})+2\left(\log f_{n}(\mathcal{V})\right)^{2} \in \mathscr{O}\left(\left(\log f_{n}(\mathcal{V})\right)^{2}\right)
$$

By Proposition 3.1 and the fact that $f_{n}\left(G_{A}\right) \leq f_{n}(\mathcal{V})$,

$$
\log f_{n}\left(Y_{A}^{1} *_{\rho} G_{A}\right) \leq \log f_{n}\left(G_{A}\right)+n \log f_{n+1}(A) \in \mathscr{O}\left(n\left(\log f_{n+1}(\mathcal{V})\right)^{2}\right),
$$

as required.

REMARK 3.3. For some particular groups $G$, it is possible to give some more precise estimations for $f_{n}(b(G))$ than the one obtained in the previous proof for a general Clifford inverse algebra $A$. For example, if $G=\mathbb{Z}_{p}$ for a prime $p$, then $G$ generates the variety $\mathcal{A}_{p}$ of Abelian groups of exponent $p$. Furthermore, it can be proved that $b\left(\mathbb{Z}_{p}\right)$ alone generates $C\left(\mathcal{A}_{p}\right)$ (as $\mathcal{A}_{p}$ coincides with the quasivariety generated by $\mathbb{Z}_{p}$, see [12]). So,

$$
f_{n}\left(b\left(\mathbb{Z}_{p}\right)\right)=\sum_{k=0}^{n}\left(\begin{array}{l}
n \\
k
\end{array}\right) \sum_{H \leq F_{k}\left(\mathcal{A}_{p}\right)}\left[F_{k}\left(\mathcal{A}_{p}\right): H\right]
$$

whereas $F_{k}\left(\mathcal{A}_{p}\right)$ is an elementary Abelian $p$-group, the $k$ th direct power of $\mathbb{Z}_{p}$. This group is in fact a $k$-dimensional vector space over the $p$-element field, so counting 
its subgroups of index $p^{k-i}$ is the same as counting its $i$-dimensional subspaces. In nearly every classical algebra textbook one can find that the number in question is the so-called Gaussian coefficient

$$
\frac{\left(p^{k}-1\right)\left(p^{k}-p\right) \ldots\left(p^{k}-p^{i-1}\right)}{\left(p^{i}-1\right)\left(p^{i}-p\right) \ldots\left(p^{i}-p^{i-1}\right)}=\frac{\left(p^{k}-1\right)\left(p^{k-1}-1\right) \ldots\left(p^{k-i+1}-1\right)}{\left(p^{i}-1\right)\left(p^{i-1}-1\right) \ldots\left(p^{1}-1\right)} \leq\left(p^{k-i+1}\right)^{i},
$$

so that

$$
\begin{aligned}
f_{n}\left(b\left(\mathbb{Z}_{p}\right)\right) & \leq \sum_{k=0}^{n}\left(\begin{array}{l}
n \\
k
\end{array}\right) \sum_{i=0}^{k} p^{i(k-i+1)} p^{k-i}=\sum_{k=0}^{n}\left(\begin{array}{l}
n \\
k
\end{array}\right) p^{k} \sum_{i=0}^{k} p^{i(k-i)} \\
& \leq \sum_{k=0}^{n}\left(\begin{array}{l}
n \\
k
\end{array}\right)(k+1) p^{\frac{1}{4} k^{2}+k} \leq(n+1)^{2} 2^{n} p^{\frac{1}{4} n^{2}+n} .
\end{aligned}
$$

Hence,

$$
\log f_{n}\left(b\left(\mathbb{Z}_{p}\right)\right) \leq \frac{\log p}{4} n^{2}+(1+\log p) n+2 \log (n+1)
$$

while (3.2) gives $\log f_{n}\left(b\left(\mathbb{Z}_{p}\right)\right) \leq 2(\log p)^{2} n^{2}+(1+\log p) n\left(\right.$ since $\left.f_{n}\left(\mathcal{A}_{p}\right)=p^{n}\right)$.

\section{A few (simple) examples}

We take the simplest example of a Clifford inverse algebra, the sink algebra of a group $G$, that is, $A=b(G)$. The corresponding semilattice $Y_{A}^{1}$ consists of a top (identity) element $\mathrm{T}$, an anti-chain of elements indexed by $G$ on the 'middle floor', and the bottom (zero) element $\infty$. Since in this case $G_{A}$ is actually $G$, to define a semidirect product $Y_{A}^{1} *_{\rho} G$ it suffices to specify a left action $\rho$ of $G$ on itself (as any automorphism of $Y_{A}^{1}$ fixes $\top$ and $\infty$ and induces a permutation of the set $G$ ).

Of course, there are many ways to do this, but we choose perhaps the most obvious one: the left translation action $\rho: g \mapsto \lambda_{g}^{\prime}$ (see Lemma 2.6). In the resulting semidirect product $Y_{A}^{1} *_{\rho} G$, we have (as is routine to verify) that the group of units is formed by pairs of the form $(\mathrm{T}, h), h \in G((\mathrm{~T}, 1)$ is the identity element of the considered monoid), while the elements of $K=\{(\infty, h): h \in G\}$ form the kernel, the unique minimal ideal. The remaining elements are pairs of the form $(g, h), g, h \in G$. Upon defining $S^{\prime}(G)=\left(Y_{A}^{1} *_{\rho} G\right) / K$, so that all elements of $K$ are collapsed into a zero element $\mathbf{0}$, the multiplication in $S^{\prime}(G)$ on the latter set of pairs works as follows:

$$
(g, h)\left(g^{\prime}, h^{\prime}\right)=\left(g \wedge h g^{\prime}, h h^{\prime}\right)= \begin{cases}\left(g, h h^{\prime}\right) & \text { if } g=h g^{\prime}, \\ \mathbf{0} & \text { otherwise } .\end{cases}
$$

Recall that the (combinatorial) Brandt semigroup $B_{X}$ over a set $X$ is the inverse semigroup defined on the set $(X \times X) \cup\{0\}$ by

$$
(x, y)\left(x^{\prime}, y^{\prime}\right)=\left\{\begin{array}{ll}
\left(x, y^{\prime}\right) & \text { if } y=x^{\prime}, \\
0 & \text { otherwise, }
\end{array} \quad \text { and }(x, y)^{-1}=(y, x),\right.
$$


for all $x, x^{\prime}, y, y^{\prime} \in X$, while 0 behaves as a zero element. Now we claim that the elements $\{(g, h): g, h \in G\} \cup\{\mathbf{0}\}$ of $S^{\prime}(G)$ form a Brandt subsemigroup of $S^{\prime}(G)$ over $G$. Indeed, it is rather straightforward to check that the mapping $\varphi$ defined by

$$
\varphi((g, h))=\left(g, h^{-1} g\right) \quad \text { and } \quad \varphi(\mathbf{0})=0
$$

is indeed an inverse semigroup isomorphism. Thus we conclude that the monoid $S^{\prime}(G)$ consists of its group of units $G$ acting on the Brandt semigroup $B_{G}$ as follows:

$$
a \cdot(g, h)=\varphi\left((\top, a)\left(g, g h^{-1}\right)\right)=\varphi\left(\left(a g, a g h^{-1}\right)\right)=(a g, h)
$$

and

$$
(g, h) \cdot a=\varphi\left(\left(g, g h^{-1}\right)(\top, a)\right)=\varphi\left(\left(g, g h^{-1} a\right)\right)=\left(g, a^{-1} h\right) .
$$

It can be proved without difficulty (although this is not essential here) that $Y_{A}^{1} *_{\rho} G$ is a subdirect product of its ideal $K \cong G$ and $S^{\prime}(G)$, so that $Y_{A}^{1} *_{\rho} G$ and $S^{\prime}(G)$ generate the same inverse monoid variety.

Similarly, one can replace the action $\rho$ by $\rho: g \mapsto \rho_{g^{-1}}^{\prime}$, the right multiplication by $g^{-1}$ extended by fixed points $T$ and $\infty$. Mutatis mutandis, this yields another semidirect product of $Y_{A}^{1}$ and $G$, again with a group kernel isomorphic to $G$, and the corresponding quotient $S(G)$ has its group of units $G$ acting on $B_{G}$ in the following way:

$$
a \cdot(g, h)=\left(g a^{-1}, h\right) \quad \text { and } \quad(g, h) \cdot a=(g, h a) .
$$

It is precisely this monoid $S(G)$, constructed for a finitely generated non-Hopfian group $G$, that was used by Sapir [23] to obtain a critical counterexample of a finitely generated inverse semigroup violating the descending chain condition for its idempotents. Also, as shown by Reilly in [21], inverse monoids of the form $S\left(\mathbb{Z}_{p}\right)$ appear as generators of minimal noncryptic inverse semigroup varieties. By another result of Reilly [22], each inverse semigroup (monoid) $S\left(\mathbb{Z}_{p}\right)$, where $p$ is a prime, generates a variety with infinitely many subvarieties, and $S\left(\mathbb{Z}_{2}\right)$ (denoted in [22] by $N_{2}$ ) is the smallest inverse semigroup with this property.

Now Corollary 2.8 immediately yields the following conclusion.

Corollary 4.1. Let $G$ be a finite nilpotent group of class $c$. Then both $\log f_{n}(S(G))$ and $\log f_{n}\left(S^{\prime}(G)\right)$ belong to the asymptotic class $\mathscr{O}\left(n^{2 c+1}\right)$.

Notice that the six-element Brandt monoid $B_{2}^{1}$ embeds into both $S(G)$ and $S^{\prime}(G)$ for each nontrivial group $G$. So, by taking $G$ to be any nontrivial finite Abelian group (for example, let $G=\mathbb{Z}_{2}$, the two-element cyclic group), we arrive at the same result as obtained by Seif [24] by other methods.

Corollary 4.2. $\log f_{n}\left(B_{2}^{1}\right) \in \mathscr{O}\left(n^{3}\right)$.

It is known that the sequence $\log f_{n}\left(B_{2}^{1}\right)$ asymptotically belongs to the interval $\left[n^{2}, n^{3}\right]$; however, the determination of the exact behavior of the free spectrum of $B_{2}^{1}$ remains an open problem that is closely connected to some rather difficult and deep 
enumeration problems on directed graphs [17]. (The analogous problem for the free spectrum of $B_{2}$ is much easier, though, see [14].)

Of course, the conclusion of Corollary 4.1 (in fact, that of Corollary 2.8) applies to finite inverse monoids with more intricate structure, such as the semidirect product $N(G)=Y_{N(G)}^{1} *_{\rho} G$, where $G$ is an arbitrary group and $\rho$ is the natural coset action defined by $\rho:(g, a N) \mapsto g a N$ for an arbitrary coset $a N$ of a normal subgroup $N$ of $G$ and $g \in G$. Further analysis would show that the latter semidirect product has $G$ as its group of units, while all other $\mathscr{D}$-classes are in a bijective correspondence (which is in fact a dual lattice isomorphism) with the lattice of all normal subgroups of $G$. A $\mathscr{D}$-class corresponding to $N \unlhd G$ turns out to have maximal subgroups isomorphic to $N$ and contains $[G: N] \mathscr{R}$ - $(\mathscr{L}$-)classes. Certainly, it would be interesting to elucidate in more detail the structure of $N(G)$ (for example, the multiplication between its elements from different $\mathscr{D}$-classes), and to characterise finite inverse monoids belonging to a variety generated by $N(G)$ for a particular finite (nilpotent) group $G$. In particular, $S^{\prime}(G)$ arises a quotient of $N(G)$ : it suffices to retain the group of units of $N(G)$ and the $\mathscr{D}$-class corresponding to the trivial (normal) subgroup of $G$, while the rest of $N(G)$ forms an ideal which can be collapsed into a zero element by a suitable Rees congruence.

\section{Acknowledgement}

The author is grateful to the anonymous referee for a meticulous reading of the manuscript and a number of useful suggestions.

\section{References}

[1] J. Berman, 'Free spectra gaps and tame congruence types', Internat. J. Algebra Comput. 5 (1995), 651-672.

[2] J. Berman and P. M. Idziak, Generative Complexity in Algebra, Memoirs of the American Mathematical Society, 175 (American Mathematical Society, Providence, RI, 2005), No. 828.

[3] S. Burris and H. P. Sankappanavar, A Course in Universal Algebra (Springer, New York, 1981).

[4] S. Crvenković and N. Ruškuc, 'Log-linear varieties of semigroups', Algebra Universalis 33 (1995), 470-474.

[5] I. Dolinka, 'On free spectra of completely regular semigroups and monoids', J. Pure Appl. Algebra 213 (2009), 1979-1990.

[6] I. Dolinka, 'On free spectra of locally testable semigroup varieties', Glasg. Math. J. 53 (2011), 623-629.

[7] I. Dolinka, 'On free spectra of finite monoids from the pseudovariety DA', Semigroup Forum 85 (2012), 244-254.

[8] E. R. Dombi and N. Ruškuc, 'On generators and presentations of semidirect products in inverse semigroups', Bull. Aust. Math. Soc. 79 (2009), 353-365.

[9] G. Grätzer and A. Kisielewicz, 'A survey of some open problems on $p_{n}$-sequences and free spectra of algebras and varieties', in: Universal Algebra and Quasigroup Theory (Jadwisin, 1989) (Heldermann, Berlin, 1992), 57-88.

[10] G. Higman, 'The orders of relatively free groups', in: Proc. Internat. Conf. Theory of Groups (Canberra, 1965) (Gordon \& Breach, New York, 1967), 153-165.

[11] D. Hobby and R. McKenzie, The Structure of Finite Algebras, Contemporary Mathematics, 76 (American Mathematical Society, Providence, RI, 1988). 
[12] M. Jackson, 'Flat algebras and the translation of universal Horn logic to equational logic', J. Symbolic Logic 73 (2008), 90-128.

[13] M. Jackson and T. Stokes, 'Agreeable semigroups', J. Algebra 266 (2003), 393-417.

[14] K. Kátai-Urbán and Cs. Szabó, 'Free spectrum of the variety generated by the five element combinatorial Brandt semigroup', Semigroup Forum 73 (2006), 253-260.

[15] K. Kátai-Urbán and Cs. Szabó, 'On the free spectrum of the variety generated by the combinatorial completely 0-simple semigroups', Glasg. Math. J. 49 (2007), 93-98.

[16] K. A. Kearnes, 'Congruence modular varieties with small free spectra', Algebra Universalis 42 (1999), 165-181.

[17] S. Kitaev and S. W. Seif, 'Word problem of the Perkins semigroup via directed acyclic graphs', Order 25 (2008), 177-194.

[18] M. V. Lawson, Inverse Semigroups: The Theory of Partial Symmetries (World Scientific, River Edge, NJ, 1998).

[19] J. Leech, 'Inverse monoids with a natural semilattice ordering', Proc. London Math. Soc. (3) 70 (1995), 146-182.

[20] P. Neumann, 'Some indecomposable varieties of groups', Quart. J. Math. Oxford (2) 14 (1965), $46-50$.

[21] N. R. Reilly, 'Minimal noncryptic varieties of inverse semigroups', Quart. J. Math. Oxford (2) 36 (1985), 467-487.

[22] N. R. Reilly, 'Large varieties generated by small inverse semigroups', Acta Sci. Math. (Szeged) 58 (1993), 25-41.

[23] M. V. Sapir, 'Identities of finite inverse semigroups', Internat. J. Algebra Comput. 3 (1993), $115-124$.

[24] S. W. Seif, 'Monoids with sub-log-exponential free spectra', J. Pure Appl. Algebra 212 (2008), $1162-1174$.

IGOR DOLINKA, Department of Mathematics and Informatics,

University of Novi Sad, Trg Dositeja Obradovića 4, 21101 Novi Sad, Serbia

e-mail: dockie@dmi.uns.ac.rs 